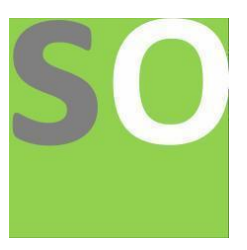

Article title: Frequency-rank Distributions in Proteomics

Authors: Raad Shaikh[1], Mrutyunjaya Behera[2]

Affiliations: Department of Physics, Indian Institute of Technology Hyderabad, IITH Main Road near NH-65, Kandi, Telangana, India[1]

Orcid ids: 0000-0002-0214-0280[1], 0000-0002-3243-5055[2]

Contact e-mail: raadshaikh2000@gmail.com

License information: This work has been published open access under Creative Commons Attribution License http://creativecommons.org/licenses/by/4.0/, which permits unrestricted use, distribution, and reproduction in any medium, provided the original work is properly cited. Conditions, terms of use and publishing policy can be found at https://www.scienceopen.com/.

Preprint statement: This article is a preprint and has not been peer-reviewed, under consideration and submitted to ScienceOpen Preprints for open peer review.

DOI: 10.14293/S2199-1006.1.SOR-.PP2VSKF.v1

Preprint first posted online: 17 June 2021

Keywords: zipf's law, power law, fat-tailed distributions, rank-size, frequency-rank distribution, statistical linguistics, bioinformatics, proteomics, protein abundance 


\title{
Frequency-rank Distributions in Proteomics
}

\author{
Raad Shaikh* Mrutyunjaya Behera \\ Indian Institute of Technology, Hyderabad, Telangana 502285
}

2021-06-17

\begin{abstract}
This paper analyzes the protein abundances in 8 organisms to determine if they fit any of a number of commonly-seen distributions in frequency-rank analyses, with the intention of drawing analogies between biochemistry and linguistics. The organisms were chosen so as to be representative and come from a wide range of body complexities. Our analysis suggests that while individual organisms fit certain distributions quite well, there is no overarching thread that unifies the protein distributions found across the living world, at least on the scale of individual proteins.
\end{abstract}

Keywords: zipf's law, power law, fat-tailed distributions, rank-size, frequency-rank distribution, statistical linguistics, bioinformatics, proteomics, protein abundance

\section{Introduction}

Proteins are the basic building block for any living organism. This family of macronutrients takes diverse forms to perform a variety of tasks essential for the growth and maintenance of biological beings [Stryer, 1981, Chap. 3]. Various kinds of proteins, all with different functions, are found in varying amounts in an organism. Here we are interested in studying the distribution of these amounts, and how much more common are more common proteins compared to rarer ones.

The way the various proteins perform their functions and give the body structure and meaning is, in a poetic sense, akin to how a book is comprised of letters and words that come together to form something beyond the sum of their parts. Each protein means something to the organism, the way each word means something to a piece of literature. It may be illuminating to take this analogy further, to explore possible similarities between artificial and natural structures by analyzing their statistical properties.

A statistical characterization of natural language was first carried out by Zipf, in his famous Zipf's law - an inverse power law between frequency and rank of words used in English literature that was found to fit almost perfectly (Powers [1998]). Since then, the model has been found in many different situations: city populations, internet traffic, and company sizes (Li [2002]). Several explanations have been offered: first-order rank expansions of various natural distributions (Belevitch [1959]), the principle of least effort (i Cancho and Solé [2003]), and preferential attachment

*Email: ep18btech11011@iith.ac.in 
(Lin et al. [2015]). However, it may be prudent to explore other, similar distributions for any of these situations rather than settling on a power law - often one may find a better fit (Clauset et al. [2009]).

This literary analogy has been explored (Mantegna et al. [1995], Som et al. [2001]) for the case of codons, the nucleotide sequences that code for amino acids (the building blocks of protein). In that case the analogy with language is somewhat more direct (see Table 1) (Crick [1970]).

Table 1: Central dogma of molecular biology

\begin{tabular}{ccc}
\hline Letter & $\Longleftrightarrow$ & Nucleotide \\
Word & $\Longleftrightarrow$ & Codon (expressed as Amino Acid) \\
Sentence & $\Longleftrightarrow$ & Gene (expressed as Protein) \\
Volume & $\Longleftrightarrow$ & Chromosome \\
Book & $\Longleftrightarrow$ & Genome \\
\hline
\end{tabular}

To our knowledge, however, this analysis has not been carried out with relative abundances of expressed proteins. A modest review of possible statistical models and distributions that can be fit to a proteome, as it is called, is undertaken here.

\section{Method}

Datasets for the relative abundance of expressed proteins in various organisms were found on https://www.proteomaps.net/, by Liebermeister et al. [2014]. Distribution fitting was carried out with the Python module lmfit (Newville et al. [2021]). lmfit provides an interface to fit a dataset to a user-defined curve using non-linear least-squares minimization. This was chosen over another popular curve-fitting module, scipy.curve_fit, because it can conveniently output various goodness-of-fit parameters.

The dataset under analysis includes, for several organisms, the abundance of each protein that the organism can express. Abundance is reported in four forms: Abundance (Original), Abundance (ppm), Size-weighted Abundance (Original), and Size-weighted Abundance (ppm). Abundance (Original) counts the number of expressed protein molecules of each type available in a typical cell of the organism. Abundance (ppm) divides this by the sum total of protein molecules in the cell. Size-weighted Abundance (original) measures the 'weight' of a particular protein in a cell, with the 'weight' referring not to mass but to the protein's length (i.e. how many amino acids it is composed of). Size-weighted Abundance (ppm) is calculated by taking this length-weighted abundance value for a particular protein, and dividing by the sum total of (length-weighted) proteins in the cell.

The second and fourth of these are analyzed in this report. Moreover, a variety of organisms are available and were utilized, from simple bacteria to a modern mammal, as summarized in Table 2. This allows for cross-comparison of the proteome distribution across various levels in the evolutionary hierarchy, and helps identify any universal trends.

The datasets were loaded into a Python script, and the relevant columns (Abundance ppm and SizeWeightedAbundance ppm) were extracted and sorted in descending order, with the intention of analyzing them separately. An integer array of appropriate size was generated to serve as the rank. 
Table 2: Organisms used in proteome analysis

\begin{tabular}{ccc}
\hline Name & Classification & Dataset Reference \\
\hline Mycoplasma pneumoniae & Bacteria (Tenericutes) & Kühner et al. [2009] \\
Escherichia coli & Proteobacteria & Lu et al. [2007] \\
Synechocystis sp. 6803 & Cyanobacteria & Wegener et al. [2010] \\
Saccharomyces cerevisiae (bakers' yeast) & Fungi & De Godoy et al. [2008] \\
Schizosaccharomyces pombe (fission yeast) & Fungi & Marguerat et al. [2012] \\
Arabidopsis thaliana & Plant (Eudicotidae) & Castellana et al. [2008] \\
Drosophila melanogaster (fruit fly) & Animal (Insecta) & Brunner et al. [2007] \\
Pan troglodytes (chimpanzee) & Animal (Primates) & Khan et al. [2013] \\
\hline
\end{tabular}

These operations were handled with the Python module numpy (Harris et al. [2020]). A number of functions and distributions were then defined, taking inspiration from similar use-cases in earlier literature; these are summarized in Table 3. Many of these find mention in (Clauset et al. [2009]).

Table 3: Summary of common rank-size distributions

\begin{tabular}{ccc}
\hline Name & Distribution & Precedent \\
\hline Zipf & $k \cdot x^{-s}$ & Powers [1998] \\
Zipf-Mandelbrot & $k \cdot(x+h)^{-s}$ & Mouillot and Lepretre [2000] \\
Stretched Exponential & $k \cdot \exp \left(-(h x)^{s}\right)$ & Elton [2018] \\
Weibull & $k \cdot(h x)^{s} \exp \left(-(h x)^{s+1}\right)$ & Elton [2018] \\
Parabolic Fractal & $k \cdot x^{-s} \exp \left(-h \ln (x)^{2}\right)$ & Laherrère [1996] \\
Power law with Exponential cutoff & $k \cdot x^{-s} \exp (-h x)$ & Clauset et al. [2009] \\
\hline
\end{tabular}

Then, Imfit was called to fit the abundance/frequency data to each distribution, as a function of rank. This module outputs certain goodness-of-fit results for each fit that it performs: chi-squared, reduced chi-squared, AIC, and BIC. It also gives parameter estimates (with optional confidence intervals), and correlations between parameters. Note that many distributions have non-trivial support for their parameters, so lmfit's options of constraining the parameters must sometimes be used. Also, often numpy will return NaN errors when evaluating too-big numbers or encountering unsupported calculations, so the choice of initial parameter guesses must be made judiciously.

Finally, fitted models were plotted against the real data with the module matplotlib.pyplot (Hunter [2007]). The goodness-of-fit results were also analyzed to choose the best-fitting model distribution in each case.

The analysis code can be found at https://github.com/raadshaikh/EP4130-project

\section{Results}

Distributions given by the functional forms in Table 3 were fitted to each organism (Table 2), for both protein Abundance and protein Size-weighted Abundance. Log-log plots of the fitted models 
superimposed on the real data scatterplot are depicted, and the curve-fitting parameters are summarized.

In the interest of brevity, the results of only three of the organisms are depicted here (M. pneumoniae, S. cerevisiae, and P. troglodytes). Results for the other organisms are available from the authors on request.

\subsection{Abundance (ppm)}

Table 4: M. pneumoniae

\begin{tabular}{c|c|ccc}
\hline & & Zipf & Zipf-Mandelbrot & Stretched exp. \\
\hline Fit Stats & (Data points) & 411 & 411 & 411 \\
& (Vars) & 2 & 3 & 3 \\
& $\chi^{2}$ & 0.87821390 & 0.05326159 & 0.06808694 \\
& Reduced $\chi^{2}$ & 0.00214722 & $1.3054 \mathrm{e}-04$ & $1.6688 \mathrm{e}-04$ \\
& AIC & -2523.01637 & -3672.91571 & -3571.98645 \\
& BIC & -2514.97918 & -3660.85993 & -3559.93067 \\
\hline Variables & $k$ & $1.46166298 \pm 0.0357$ & $25.8527967 \pm 2.2258$ & $1.94237807 \pm 0.0574$ \\
& $s$ & $0.63203333 \pm 0.0096$ & $1.30052042 \pm 0.0194$ & $0.39984996 \pm 0.0075$ \\
& $h$ & & $10.8210107 \pm 0.3949$ & $0.23059136 \pm 0.0170$ \\
\hline
\end{tabular}

Table 5: M. pneumoniae (contd.)

\begin{tabular}{c|c|ccc}
\hline & & Weibull & Parabolic fractal & Cutoff Power law \\
\hline Fit Stats & (Data points) & 411 & 411 & 411 \\
& (Vars) & 3 & 3 & 3 \\
& $\chi^{2}$ & 0.52503971 & 0.04267558 & 0.15580771 \\
& Reduced $\chi^{2}$ & 0.00128686 & $1.0460 \mathrm{e}-04$ & $3.8188 \mathrm{e}-04$ \\
& AIC & -2732.44146 & -3763.98859 & -3231.74533 \\
& $\mathrm{BIC}$ & -2720.38568 & -3751.93281 & -3219.68955 \\
\hline Variables & $k$ & $0.85290897 \pm 0.0257$ & $1.02688318 \pm 0.0086$ & $1.19998563 \pm 0.0156$ \\
& $s$ & $1.0000 \mathrm{e}-04 \pm 0.0163$ & $0.01446800 \pm 0.0083$ & $0.35524599 \pm 0.0084$ \\
& $h$ & $0.04316303 \pm 0.0012$ & $0.13255106 \pm 0.0018$ & $0.01397561 \pm 5.0888 \mathrm{e}-04$ \\
\hline
\end{tabular}


Table 6: S. cerevisiae

\begin{tabular}{c|c|ccc}
\hline & & Zipf & Zipf-Mandelbrot & Stretched exp. \\
\hline Fit Stats & (Data points) & 4033 & 4033 & 4033 \\
& (Vars) & 2 & 3 & 3 \\
& $\chi^{2}$ & 0.91021542 & 0.09319177 & 0.10965769 \\
& Reduced $\chi^{2}$ & $2.2580 \mathrm{e}-04$ & $2.3125 \mathrm{e}-05$ & $2.7210 \mathrm{e}-05$ \\
& AIC & -33858.4383 & -43047.7337 & -42391.5474 \\
& $\mathrm{BIC}$ & -33845.8338 & -43028.8269 & -42372.6406 \\
\hline Variables & $k$ & $1.4715 \pm 0.0114$ & $9.3523 \pm 0.1828$ & $3.4518 \pm 0.0682$ \\
& $s$ & $0.6780 \pm 0.0027$ & $1.0691 \pm 0.0044$ & $0.2676 \pm 0.0022$ \\
& $h$ & & $6.8715 \pm 0.0940$ & $1.5494 \pm 0.0933$ \\
\hline
\end{tabular}

Table 7: S. cerevisiae (contd.)

\begin{tabular}{c|c|ccc}
\hline & & Weibull & Parabolic fractal & Cutoff Power law \\
\hline Fit Stats & (Data points) & 4033 & 4033 & 4033 \\
& (Vars) & 3 & 3 & 3 \\
& $\chi^{2}$ & 0.93604964 & 0.08232613 & 0.21895082 \\
& Reduced $\chi^{2}$ & $2.3227 \mathrm{e}-04$ & $2.0428 \mathrm{e}-05$ & $5.4330 \mathrm{e}-05$ \\
& AIC & -33743.5659 & -43547.7081 & -39602.7944 \\
& BIC & -33724.6591 & -43528.8013 & -39583.8876 \\
\hline Variables & $k$ & $0.7794 \pm 0.0106$ & $1.0539 \pm 0.0037$ & $1.2172 \pm 0.0058$ \\
& $s$ & $1.0000 \mathrm{e}-04 \pm 0.00735504$ & $0.1558 \pm 0.0032$ & $0.4608 \pm 0.0026$ \\
& $h$ & $0.04136502 \pm 5.6235 \mathrm{e}-04$ & $0.09501410 \pm 6.4584 \mathrm{e}-04$ & $0.00561233 \pm 9.6514 \mathrm{e}-05$ \\
\hline
\end{tabular}

Table 8: P. troglodytes

\begin{tabular}{c|c|ccc}
\hline & & Zipf & Zipf-Mandelbrot & Stretched exp. \\
\hline Fit Stats & (Data points) & 3389 & 3389 & 3389 \\
& (Vars) & 2 & 3 & 3 \\
& $\chi^{2}$ & 0.08345137 & 0.07169910 & 0.04899726 \\
& Reduced $\chi^{2}$ & $2.4639 \mathrm{e}-05$ & $2.1175 \mathrm{e}-05$ & $1.4471 \mathrm{e}-05$ \\
& AIC & -35959.3269 & -36471.7295 & -37761.9688 \\
& $\mathrm{BIC}$ & -35947.0704 & -36453.3447 & -37743.5839 \\
\hline Variables & $k$ & $1.060280 \pm 0.003799$ & $1.282463 \pm 0.012298$ & $3676.557 \pm 813.4533$ \\
& $s$ & $0.684399 \pm 0.001333$ & $0.725658 \pm 0.002423$ & $0.067413 \pm 0.001490$ \\
& $h$ & & $0.423357 \pm 0.021614$ & $3.8603 \mathrm{e}+13 \pm 4.1942 \mathrm{e}+13$ \\
\hline
\end{tabular}


Table 9: P. troglodytes (contd.)

\begin{tabular}{c|c|ccc}
\hline & & Weibull & Parabolic fractal & Cutoff Power law \\
\hline Fit Stats & (Data points) & 3389 & 3389 & 3389 \\
& (Vars) & 3 & 3 & 3 \\
& $\chi^{2}$ & 0.99436500 & 0.05158171 & 0.02248416 \\
& Reduced $\chi^{2}$ & $2.9367 \mathrm{e}-04$ & $1.5234 \mathrm{e}-05$ & $6.6403 \mathrm{e}-06$ \\
& AIC & -27559.9264 & -37587.7648 & -40401.8410 \\
& BIC & -27541.5415 & -37569.3799 & -40383.4561 \\
\hline Variables & $k$ & $0.53419504 \pm 0.01348427$ & $0.97473202 \pm 0.00341986$ & $0.99201327 \pm 0.00204308$ \\
& $s$ & $1.0000 \mathrm{e}-04 \pm 0.00997017$ & $0.55542340 \pm 0.00314036$ & $0.61982417 \pm 9.6321 \mathrm{e}-04$ \\
& $h$ & $0.05087876 \pm 0.00126161$ & $0.02153213 \pm 5.3461 \mathrm{e}-04$ & $7.7856 \mathrm{e}-04 \pm 1.2436 \mathrm{e}-05$ \\
\hline
\end{tabular}

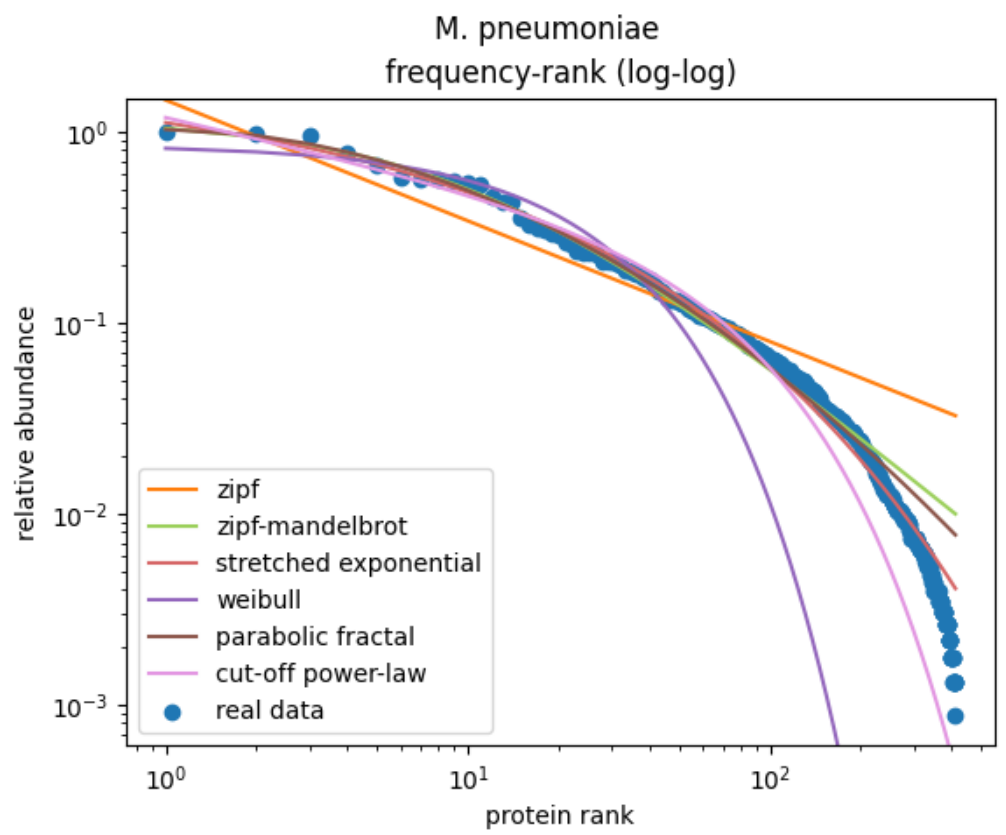

Figure 1: M. pneumoniae fitted models (Abundance) 


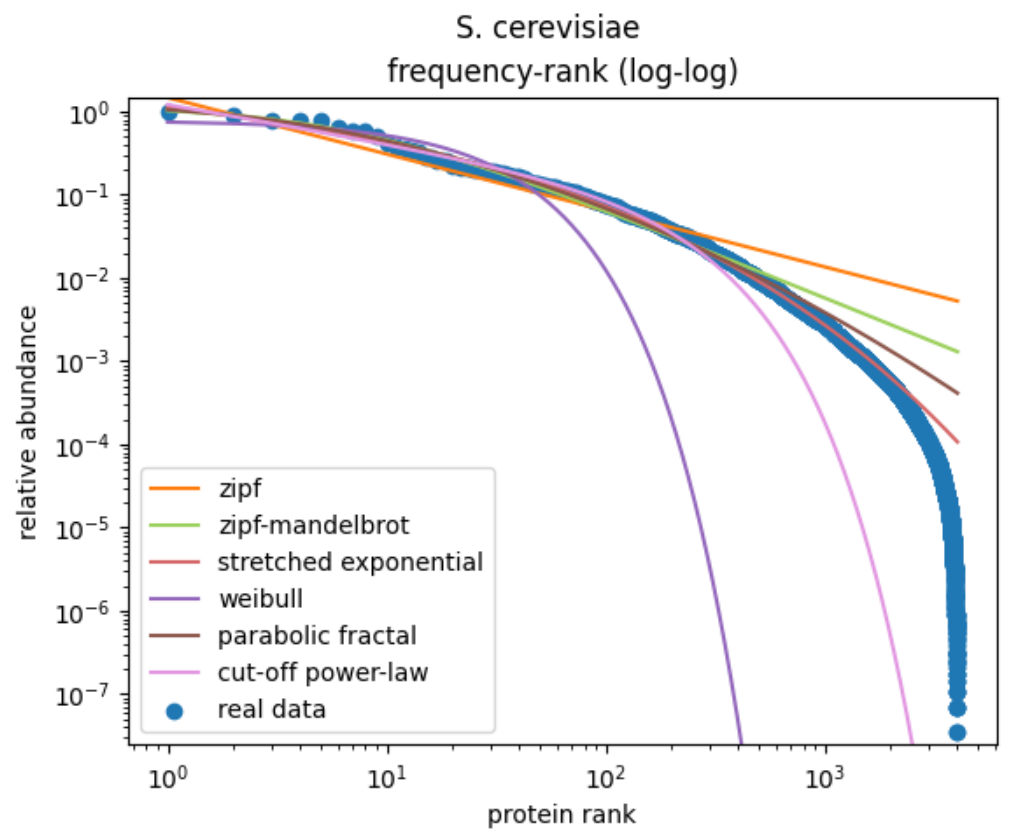

Figure 2: S. cerevisiae fitted models (Abundance)

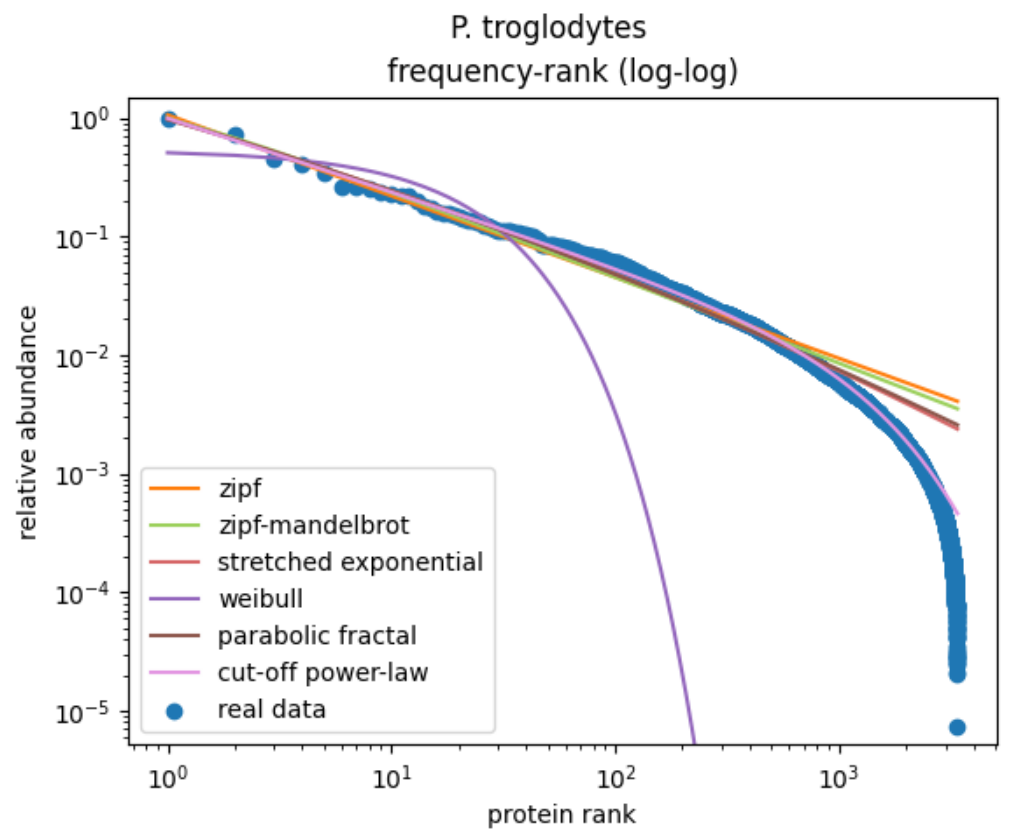

Figure 3: P. troglodytes fitted models (Abundance) 


\section{$3.2 \quad$ Size-weighted abundance (ppm)}

Table 10: M. pneumoniae

\begin{tabular}{c|c|ccc}
\hline & & Zipf & Zipf-Mandelbrot & Stretched exp. \\
\hline Fit Stats & (Data points) & 411 & 411 & 411 \\
& (Vars) & 2 & 3 & 3 \\
& $\chi^{2}$ & 0.45440326 & 0.04060832 & 0.04046302 \\
& Reduced $\chi^{2}$ & 0.00111101 & $9.9530 \mathrm{e}-05$ & $9.9174 \mathrm{e}-05$ \\
& AIC & -2793.82638 & -3784.39631 & -3785.86953 \\
& $\mathrm{BIC}$ & -2785.78920 & -3772.34053 & -3773.81375 \\
\hline Variables & $k$ & $1.2926 \pm 0.0264$ & $11.9762 \pm 0.8579$ & $2.3996 \pm 0.0892$ \\
& $s$ & $0.6733 \pm 0.0088$ & $1.2195 \pm 0.0174$ & $0.3430 \pm 0.0067$ \\
& $h$ & & $6.8282 \pm 0.2639$ & $0.6058 \pm 0.0598$ \\
\hline
\end{tabular}

Table 11: M. pneumoniae (contd.)

\begin{tabular}{c|c|ccc}
\hline & & Weibull & Parabolic fractal & Cutoff Power law \\
\hline Fit Stats & (Data points) & 411 & 411 & 411 \\
& (Vars) & 3 & 3 & 3 \\
& $\chi^{2}$ & 0.41348418 & 0.03454363 & 0.08244674 \\
& Reduced $\chi^{2}$ & 0.00101344 & $8.4666 \mathrm{e}-05$ & $2.0208 \mathrm{e}-04$ \\
& AIC & -2830.61071 & -3850.87556 & -3493.33453 \\
& BIC & -2818.55493 & -3838.81978 & -3481.27876 \\
\hline Variables & $k$ & $0.7951 \pm 0.0265$ & $0.9868 \pm 0.0080$ & $1.1090 \pm 0.0117$ \\
& $s$ & $1.0000 \mathrm{e}-04 \pm 0.00210234$ & $0.1400 \pm 0.0088$ & $0.4282 \pm 0.0075$ \\
& $h$ & $0.0566 \pm 0.0018$ & $0.1200 \pm 0.0020$ & $0.01351079 \pm 5.0345 \mathrm{e}-04$ \\
\hline
\end{tabular}

Table 12: S. cerevisiae

\begin{tabular}{c|c|ccc}
\hline & & Zipf & Zipf-Mandelbrot & Stretched exp. \\
\hline Fit Stats & (Data points) & 4033 & 4033 & 4033 \\
& (Vars) & 2 & 3 & 3 \\
& $\chi^{2}$ & 1.36812649 & 0.02379189 & 0.07601027 \\
& Reduced $\chi^{2}$ & $3.3940 \mathrm{e}-04$ & $5.9037 \mathrm{e}-06$ & $1.8861 \mathrm{e}-05$ \\
& AIC & -32214.9252 & -48554.0476 & -43869.6222 \\
& $\mathrm{BIC}$ & -32202.3207 & -48535.1408 & -43850.7154 \\
\hline Variables & $k$ & $1.5435 \pm 0.0140$ & $49.8273 \pm 1.0039$ & $1.7428 \pm 0.0151$ \\
& $s$ & $0.6863 \pm 0.0033$ & $1.4267 \pm 0.0043$ & $0.4272 \pm 0.0025$ \\
& $h$ & & $14.4637 \pm 0.1014$ & $0.1711 \pm 0.0035$ \\
\hline
\end{tabular}


Table 13: S. cerevisiae (contd.)

\begin{tabular}{c|c|ccc}
\hline & & Weibull & Parabolic fractal & Cutoff Power law \\
\hline Fit Stats & (Data points) & 411 & 411 & 411 \\
& (Vars) & 3 & 3 & 3 \\
& $\chi^{2}$ & 0.39730644 & 0.03225122 & 0.18381292 \\
& Reduced $\chi^{2}$ & $9.8587 \mathrm{e}-05$ & $8.0028 \mathrm{e}-06$ & $4.5611 \mathrm{e}-05$ \\
& AIC & -37199.6881 & -47327.1639 & -40308.2816 \\
& BIC & -37180.7813 & -47308.2571 & -40289.3748 \\
\hline Variables & $k$ & $0.8628 \pm 0.0070$ & $0.9790 \pm 0.0023$ & $1.1477 \pm 0.0053$ \\
& $s$ & $1.0000 \mathrm{e}-04 \pm 6.7861 \mathrm{e}-04$ & $-0.0387 \pm 0.0022$ & $0.2920 \pm 0.0031$ \\
& $h$ & $0.04191958 \pm 3.3765 \mathrm{e}-04$ & $0.13972537 \pm 4.8401 \mathrm{e}-04$ & $0.01810041 \pm 2.0528 \mathrm{e}-04$ \\
\hline
\end{tabular}

Table 14: P. troglodytes

\begin{tabular}{c|c|ccc}
\hline & & Zipf & Zipf-Mandelbrot & Stretched exp. \\
\hline Fit Stats & (Data points) & 3389 & 3389 & 3389 \\
& (Vars) & 2 & 3 & 3 \\
& $\chi^{2}$ & 0.15956443 & 0.10919979 & 0.05511395 \\
& Reduced $\chi^{2}$ & $4.7111 \mathrm{e}-05$ & $3.2250 \mathrm{e}-05$ & $1.6277 \mathrm{e}-05$ \\
& $\mathrm{AIC}$ & -33762.6324 & -35045.9739 & -37363.2915 \\
& $\mathrm{BIC}$ & -33750.3758 & -35027.5890 & -37344.9066 \\
\hline Variables & $k$ & $1.0968 \pm 0.0050$ & $1.6176 \pm 0.0191$ & $66.6956 \pm 5.4221$ \\
& $s$ & $0.6484 \pm 0.0015$ & $0.7271 \pm 0.0027$ & $0.1067 \pm 0.0014$ \\
& $h$ & & $1.1021 \pm 0.0376$ & $788974.675 \pm 282950.209$ \\
\hline
\end{tabular}

Table 15: P. troglodytes (contd.)

\begin{tabular}{c|c|ccc}
\hline & & Weibull & Parabolic fractal & Cutoff Power law \\
\hline Fit Stats & (Data points) & 3389 & 3389 & 3389 \\
& (Vars) & 3 & 3 & 3 \\
& $\chi^{2}$ & 1.12113164 & 0.06283341 & 0.02247501 \\
& Reduced $\chi^{2}$ & $3.3111 \mathrm{e}-04$ & $1.8557 \mathrm{e}-05$ & $6.6376 \mathrm{e}-06$ \\
& AIC & -27153.2820 & -36919.0477 & -40403.2213 \\
& BIC & -27134.8971 & -36900.6629 & -40384.8364 \\
\hline Variables & $k$ & $0.4014 \pm 0.0092$ & $0.9426 \pm 0.0036$ & $0.9911 \pm 0.0019$ \\
& $s$ & $1.0000 \mathrm{e}-04 \pm 0.00972963$ & $0.4450 \pm 0.0031$ & $0.56081190 \pm 8.4955 \mathrm{e}-04$ \\
& $h$ & $0.02282832 \pm 5.3103 \mathrm{e}-04$ & $0.03227153 \pm 5.1270 \mathrm{e}-04$ & $9.7006 \mathrm{e}-04 \pm 1.0672 \mathrm{e}-05$ \\
\hline
\end{tabular}




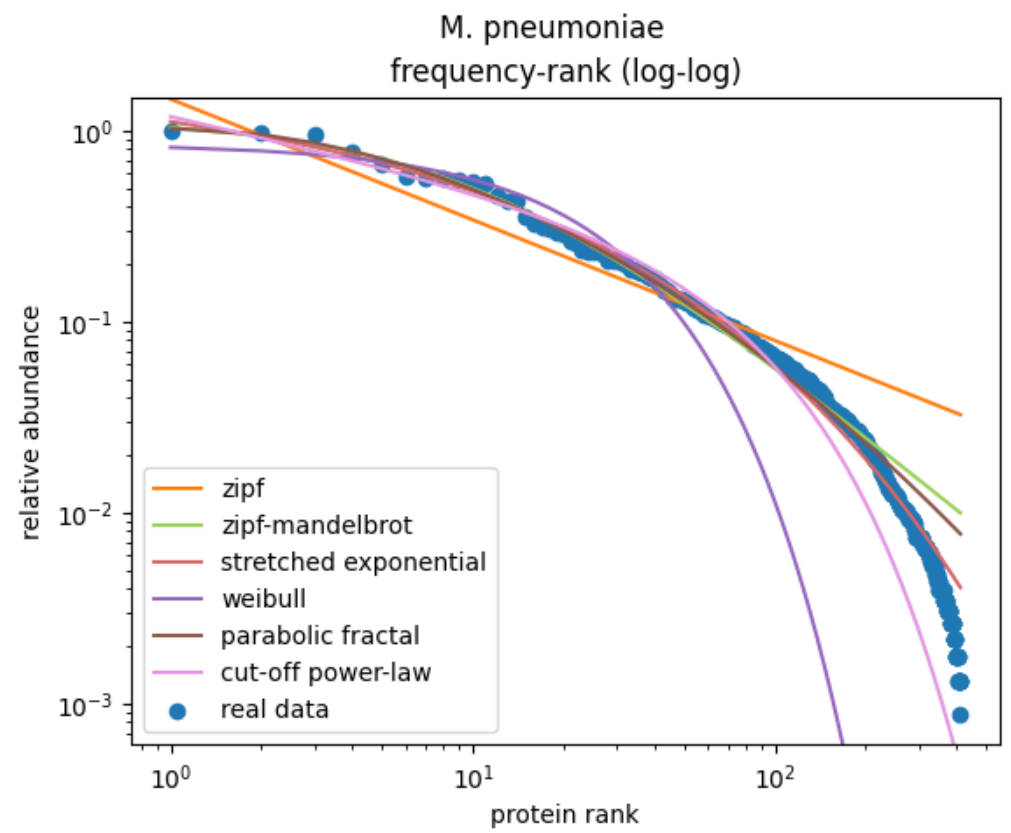

Figure 4: M. pneumoniae fitted models (Size-weighted Abundance)

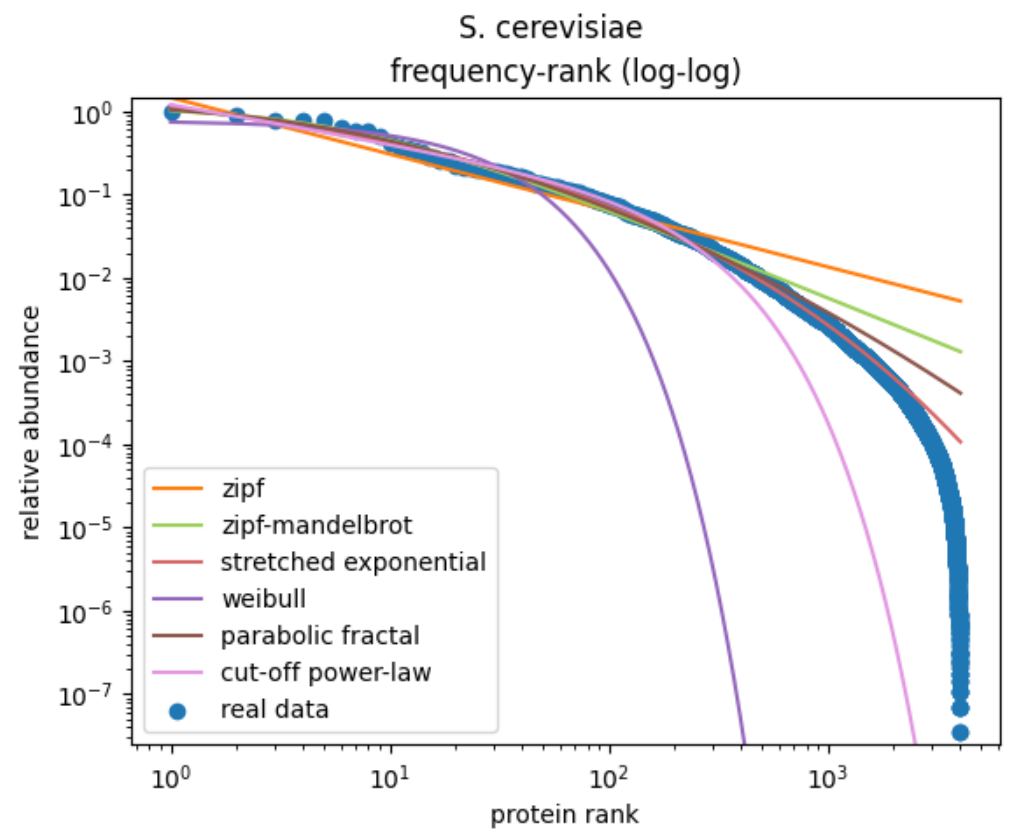

Figure 5: S. cerevisiae fitted models (Size-weighted Abundance) 


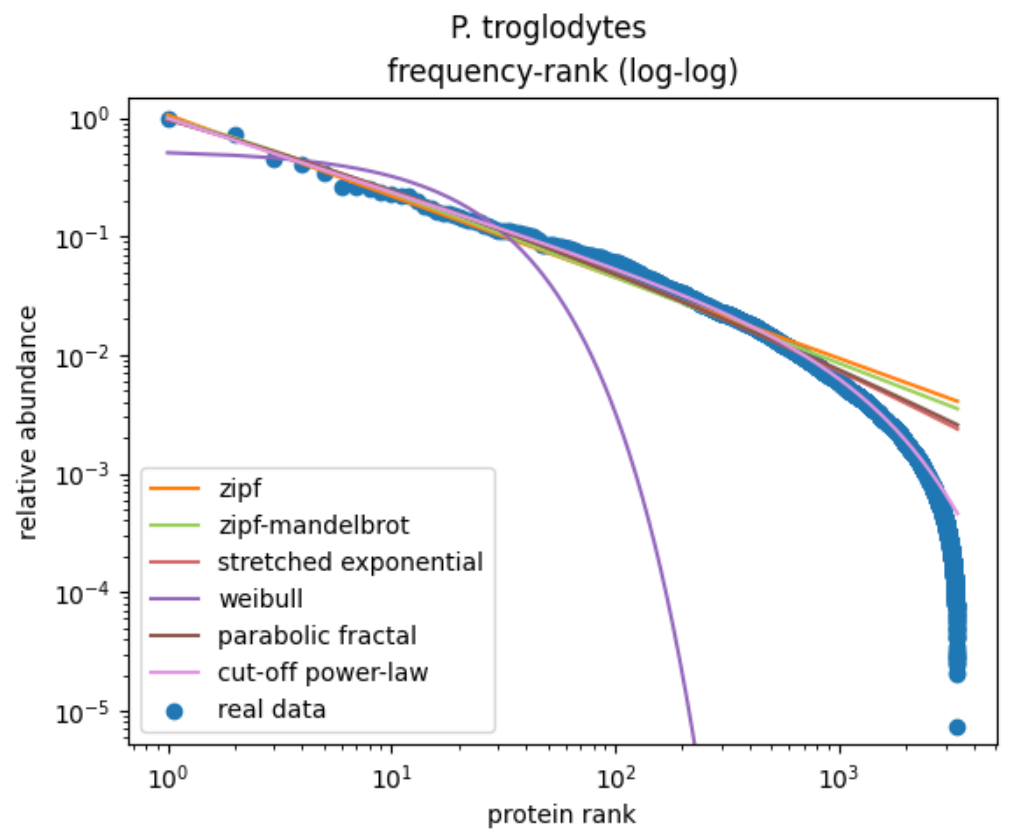

Figure 6: P. troglodytes fitted models (Size-weighted Abundance)

\section{Discussion}

We now wish to select the best model for a variety of cases. This selection may be done by information-theoretic criteria (AIC and BIC), or by frequentist methods $\left(\chi^{2}\right.$ and reduced $\chi^{2}$ ).

In the information-theoretic approach, the 'best' model is selected as that with the smallest $\mathrm{AIC}$ or BIC value; and the difference between the AIC/BIC value of some particular model and the minimum value determines how seriously we can consider that model as an alternative. The selection rules for this criteria are rather subjective (Shi et al. [2012]), but it is agreed that a difference of more than 10 all but rules out the alternative models. This is indeed the case in 15 out of the 16 datasets that were analyzed, indicating that in most cases, the best fitting model is the only one that is considerable.

The sole exception is Size-weighted Abundance in E. coli, where the second-best model has 'substantial support' by AIC, but there is 'some positive evidence against' it by BIC. In any case, this may be considered an outlier in this regard.

As for the frequentist methods, there are some problems. First of all, the analysis of $\chi^{2}$ and related statistics is only really valid when the models are linear in the parameters, which is not the case here. Thus, any interpretation here must be done with great caution. (Andrae et al. [2010]) Now, for a good fit, we would want to find reduced $\chi^{2}$ to be close to 1 , but in all cases here it is much smaller, nearly close to 0 . This may be indicative of errors or uncertainty in the data being grossly overestimated. Thus, to take these values on their own merit and interpret them absolutely may be dubious.

Since these problems are more or less the same across the datasets, we can make the (rather weak) claim that models with less reduced $\chi^{2}$ are better fits, but no more than that. Going by this rule of thumb, we find the frequentist model selection to agree with the information-theoretic approach in all cases. 
With these rules for selecting models, the best-fitting (and second-best) distribution for each dataset can be summarized as in Table 16:

\begin{tabular}{c|c|c} 
& Abundance & Size-weighted Abundance \\
\hline M. pneumoniae & Parab. frac., Zipf-Mandelbrot & Parab. frac., Zipf-Mandelbrot \\
E. coli & Cutoff Powerlaw, Stretched exp. & Stretched exp., Parab. frac. \\
Syn. sp. 6803 & Cutoff Powerlaw, Stretched exp. & Cutoff Powerlaw, Stretched exp. \\
S. cerevisiae & Parab. frac., Zipf-Mandelbrot & Zipf-Mandelbrot, Parab. frac. \\
S. pombe & Parab. frac., Stretched exp. & Parab. frac., Zipf-Mandelbrot \\
A. thaliana & Stretched exp., Parab. frac. & Parab. frac., Stretched exp. \\
D. melanogaster & Stretched exp., Parab. frac. & Parab. frac., Stretched exp. \\
P. troglodytes & Cutoff Powerlaw, Stretched exp. & Cutoff Powerlaw, Stretched exp. \\
\hline
\end{tabular}

Table 16: Best, and second-best models for each dataset

The null hypothesis for this analysis is that there will be no clear trends across the data, and that there is no one distribution that models all protein frequencies well.

The alternate hypotheses of this analysis are:

- Is there a single distribution that best models all organisms?

- Are there any trends in terms of the distribution as we move up in the evolutionary hierarchy?

- Are Abundance and Size-weighted Abundance distributed the same way, for the same organism?

We can see that the most common well-fitting distributions are Parabolic Fractal, Stretched Exponential, and Cut-off Power-law, but these are more or less strewn randomly across the organisms. It may also be noted that in Size-weighted Abundance, the Parabolic Fractal is slightly more common than others.

Apart from this, no clear trends can be identified with respect to any of our hypotheses. Thus we cannot quite reject our null hypothesis, and conclude that there is no method to the madness of proteomics that can be identified yet.

This report analyzes frequency-rank distributions at the level of expressed proteins found in cells. Future avenues of research could involve moving up or down a level of organization. At a lower level, we can analyze the distributions in codon frequency (as in Kim et al. [2005]) or aminoacid frequency. At a higher level, we can cluster proteins of similar function at various functional levels (as can be seen in the Proteomaps of Liebermeister et al. [2014]), e.g. proteins that are involved with metabolism; more specifically, energy metabolism; even more specifically, oxidative phosphorylation, etc.

\section{Acknowledgements}

This research was carried out at the Indian Institute of Technology, Hyderabad. We are grateful to the Government of India for developing this institute and subsidizing the authors' education here. 
We would also like to thank

- Dr. Shantanu Desai, for mentoring us on statistical analysis and supervising this project

- Razia Shaikh, for suggesting the topic of the paper, providing an accessible introduction to relevant topics in biochemistry, and pointing out useful datasets in proteomics

- Alexandra Elbakyan, for indirect but invaluable assistance during the course of our research The authors declare no conflict of interest.

\section{References}

Lubert. Stryer. Biochemistry / Lubert Stryer. W. H. Freeman, San Francisco, 2d ed. edition, 1981. ISBN 0716712261.

David M. W. Powers. Applications and explanations of Zipf's law. In New Methods in Language Processing and Computational Natural Language Learning, 1998. URL https://www.aclweb. org/anthology/w98-1218.

Wentian Li. Zipf's law everywhere. Glottometrics, 5:14-21, 2002.

Vitold Belevitch. On the statistical laws of linguistic distributions. In Annales de la Societe Scientifique de Bruxelles, volume 73, pages 301-326, 1959.

Ramon Ferrer i Cancho and Ricard V Solé. Least effort and the origins of scaling in human language. Proceedings of the National Academy of Sciences, 100(3):788-791, 2003.

Ruokuang Lin, Qianli D. Y. Ma, and Chunhua Bian. Scaling laws in human speech, decreasing emergence of new words and a generalized model, 2015.

Aaron Clauset, Cosma Rohilla Shalizi, and Mark EJ Newman. Power-law distributions in empirical data. SIAM review, 51(4):661-703, 2009.

RN Mantegna, SV Buldyrev, AL Goldberger, S Havlin, C-K Peng, M Simons, and HE Stanley. Systematic analysis of coding and noncoding dna sequences using methods of statistical linguistics. Physical Review E, 52(3):2939, 1995.

A Som, S Chattopadhyay, J Chakrabarti, and D Bandyopadhyay. Codon distributions in dna. Physical Review E, 63(5):051908, 2001.

Francis Crick. Central dogma of molecular biology. Nature, 227(5258):561-563, 1970.

Wolfram Liebermeister, Elad Noor, Avi Flamholz, Dan Davidi, Jörg Bernhardt, and Ron Milo. Visual account of protein investment in cellular functions. Proceedings of the National Academy of Sciences, 111(23):8488-8493, 2014.

Matt Newville, Renee Otten, Andrew Nelson, Antonino Ingargiola, Till Stensitzki, Dan Allan, Austin Fox, Faustin Carter, Michał, Dima Pustakhod, lneuhaus, Sebastian Weigand, Ray Osborn, Glenn, Christoph Deil, Mark, Allan L. R. Hansen, Gustavo Pasquevich, Leon Foks, Nicholas Zobrist, Oliver Frost, Alexandre Beelen, Stuermer, kwertyops, Anthony Polloreno, Shane Caldwell, Anthony Almarza, Arun Persaud, Ben Gamari, and Benjamin F. Maier. lmfit/lmfit-py 1.0.2, February 2021. URL https://doi.org/10.5281/zenodo. 4516651. 
Sebastian Kühner, Vera van Noort, Matthew J Betts, Alejandra Leo-Macias, Claire Batisse, Michaela Rode, Takuji Yamada, Tobias Maier, Samuel Bader, Pedro Beltran-Alvarez, et al. Proteome organization in a genome-reduced bacterium. Science, 326(5957):1235-1240, 2009.

Peng Lu, Christine Vogel, Rong Wang, Xin Yao, and Edward M Marcotte. Absolute protein expression profiling estimates the relative contributions of transcriptional and translational regulation. Nature biotechnology, 25(1):117-124, 2007.

Kimberly M Wegener, Abhay K Singh, Jon M Jacobs, Thanura Elvitigala, Eric A Welsh, Nir Keren, Marina A Gritsenko, Bijoy K Ghosh, David G Camp II, Richard D Smith, et al. Global proteomics reveal an atypical strategy for carbon/nitrogen assimilation by a cyanobacterium under diverse environmental perturbations. Molecular \&s Cellular Proteomics, 9(12):2678-2689, 2010 .

Lyris MF De Godoy, Jesper V Olsen, Jürgen Cox, Michael L Nielsen, Nina C Hubner, Florian Fröhlich, Tobias C Walther, and Matthias Mann. Comprehensive mass-spectrometry-based proteome quantification of haploid versus diploid yeast. Nature, 455(7217):1251-1254, 2008.

Samuel Marguerat, Alexander Schmidt, Sandra Codlin, Wei Chen, Ruedi Aebersold, and Jürg Bähler. Quantitative analysis of fission yeast transcriptomes and proteomes in proliferating and quiescent cells. Cell, 151(3):671-683, 2012.

Natalie E Castellana, Samuel H Payne, Zhouxin Shen, Mario Stanke, Vineet Bafna, and Steven P Briggs. Discovery and revision of arabidopsis genes by proteogenomics. Proceedings of the national academy of sciences, 105(52):21034-21038, 2008.

Erich Brunner, Christian H Ahrens, Sonali Mohanty, Hansruedi Baetschmann, Sandra Loevenich, Frank Potthast, Eric W Deutsch, Christian Panse, Ulrik de Lichtenberg, Oliver Rinner, et al. A high-quality catalog of the drosophila melanogaster proteome. Nature biotechnology, 25(5): 576-583, 2007.

Zia Khan, Michael J Ford, Darren A Cusanovich, Amy Mitrano, Jonathan K Pritchard, and Yoav Gilad. Primate transcript and protein expression levels evolve under compensatory selection pressures. Science, 342(6162):1100-1104, 2013.

Charles R. Harris, K. Jarrod Millman, St'efan J. van der Walt, Ralf Gommers, Pauli Virtanen, David Cournapeau, Eric Wieser, Julian Taylor, Sebastian Berg, Nathaniel J. Smith, Robert Kern, Matti Picus, Stephan Hoyer, Marten H. van Kerkwijk, Matthew Brett, Allan Haldane, Jaime Fern'andez del R'1o, Mark Wiebe, Pearu Peterson, Pierre G'erard-Marchant, Kevin Sheppard, Tyler Reddy, Warren Weckesser, Hameer Abbasi, Christoph Gohlke, and Travis E. Oliphant. Array programming with NumPy. Nature, 585(7825):357-362, September 2020. doi: 10.1038/s41586-020-2649-2. URL https://doi .org/10.1038/s41586-020-2649-2.

David Mouillot and Alain Lepretre. Introduction of relative abundance distribution (rad) indices, estimated from the rank-frequency diagrams ( $\mathrm{rfd}$ ), to assess changes in community diversity. Environmental monitoring and assessment, 63(2):279-295, 2000.

Daniel C Elton. Stretched exponential relaxation. arXiv preprint arXiv:1808.00881, 2018.

Jean Laherrère. Distributions de type fractal parabolique dans la nature. Comptes rendus de l'Académie des sciences. Série 2. Sciences de la terre et des planètes, 322(7):535-541, 1996. 
J. D. Hunter. Matplotlib: A 2d graphics environment. Computing in Science $\&$ Engineering, 9(3): 90-95, 2007. doi: 10.1109/MCSE.2007.55.

Ke Shi, YF Huang, and Tan Lu. A comprehensive comparison of cosmological models from the latest observational data. Monthly Notices of the Royal Astronomical Society, 426(3):2452-2462, 2012 .

Rene Andrae, Tim Schulze-Hartung, and Peter Melchior. Dos and don'ts of reduced chi-squared. arXiv preprint arXiv:1012.3754, 2010.

JK Kim, SI Yang, YH Kwon, and Eun Il Lee. Codon and amino-acid distribution in dna. Chaos, Solitons \& Fractals, 23(5):1795-1807, 2005. 